\title{
Photoexcitation of graphene with twisted light
}

\author{
M. B. Farías, G. F. Quinteiro, and P. I. Tamborenea \\ Departamento de Física e IFIBA, FCEN, \\ Universidad de Buenos Aires, Ciudad Universitaria, \\ Pab. I, C1428EHA Buenos Aires, Argentina
}

\begin{abstract}
We study theoretically the interaction of twisted light with graphene. The light-matter interaction matrix elements between the tight-binding states of electrons in graphene are determined near the Dirac points. We examine the dynamics of the photoexcitation process by posing the equations of motion of the density matrix and working up to second order in the field. The time evolution of the angular momentum of the photoexcited electrons and their associated photocurrents are examined in order to elucidate the mechanisms of angular momentum transfer. We find that the transfer of spin and orbital angular momentum from light to the electrons is more akin here to the case of intraband than of interband transitions in semiconductors, due to the fact that the two relevant energy bands of graphene originate from the same atomic orbitals.

PACS numbers: 78.67.Wj, 42.50.Tx
\end{abstract}

Keywords: graphene, twisted light, orbital angular momentum

\section{INTRODUCTION}

Due to its low dimensionality and particular crystalline structure, graphene presents an unusual semi-metallic behavior, and its low-energy excitations behave as massless Dirac fermions. $\stackrel{1,2}{\text { Because }}$ of this, graphene shows unusual transport properties, like an anomalous quantum hall effect ${ }^{3}$ and Klein tunnelling,$\underline{4}+\underline{5}$ Its optical properties are also peculiar: despite being one-atom thick, graphene absorbs a significant amount of white light, and its transparency is governed by the fine structure constant, usually associated with quantum electrodynamics rather than condensed matter physics $\underline{\underline{6} .7}$

In parallel to these discoveries, a new branch of optics, the study of phase-structured light, developed vigorously in the last twenty years. The generation and applications of light carrying orbital angular momentum (OAM) or twisted light (TL) gained great attention after the seminal work of Allen et al., $\frac{8}{,}$ who showed that light carrying an integer amount of orbital angular momentum ( $\hbar \ell$, with $\ell$ an integer) may be generated in the laboratory using conventional laser beams. Current efforts in this field are directed, on the one hand, to the understanding and generation of twisted 
light beams, and, on the other hand, to the study of interaction with mesoscopic particles, atoms and molecules, and Bose-Einstein condensates. $\underline{\underline{9}}$ Only recently the interaction with solid-state systems has been studied, subject that appears to be a promising field of research and technology. Theoretical works study the interaction of TL with various semicondunctor systems $\underline{\underline{10}} \underline{-12,15,16}$ Also experiments on the field are starting to be conducted. 17,18

The interaction of graphene with light has been studied theoretically with different approaches, for instance by the calculation of the opacity and the optical conductivity,$\underset{7}{ }$ or control of photocurrents $\frac{19}{}$ The study of the interaction of graphene with light carrying OAM is interesting by itself, since it leads to questions rarely formulated in works studying the optical properties of graphene. Since the twisted light has orbital angular momentum, one may expect a transfer of OAM from the photons to the electrons in graphene. However, the analysis is complicated by the fact that the low-lying excitations of graphene are Dirac fermions, whose OAM is not well-defined. Nevertheless, there is another angular momentum, known as pseudospin associated with the honeycomb lattice of graphene, and the total angular momentum (orbital plus pseudospin) is conserved.

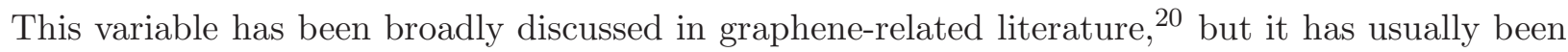
left aside in works studying interaction with light.

In this work, we study theoretically the interaction of graphene with twisted light and calculate relevant physical magnitudes: the photo-induced electric currents and the transfer of angular momentum. To this end, we first obtain the light-matter matrix elements for twisted light and graphene, and then use quantum-kinetic equations of motion to obtain the time evolution of physical observables: $\underline{21-24}$

The paper is organized as follows. In Section 【we describe the electronic states of graphene. Next, in Section III we study the light-matter interaction Hamiltonian. Section IV contains the calculation of the interaction matrix elements. In Section $\nabla$ we write the equations of motion for the density matrix and study them in the low excitation regime. Relevant physical quantities are examined in Section VI. Finally, conclusions are presented in the last Section.

\section{LOW-EXCITATION STATES IN GRAPHENE}

In Fig. 1 (left) we show the crystalline structure of graphene: a honeycomb lattice formed by carbon atoms separated by a distance $a \approx 1.42 \AA$. The first Brillouin zone is shown in Fig. 1 (right). In the tight-binding model (with nearest-neighbour hopping $t$ ) the Hamiltonian matrix is given by 


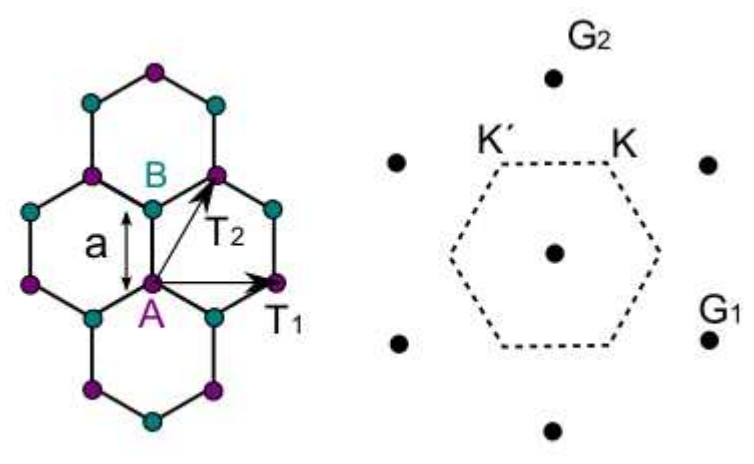

FIG. 1: Crystal structure of graphene in the direct (left) and reciprocal (right) space. $\mathbf{T}_{1}$ and $\mathbf{T}_{2}$ are the primitive vectors of the Bravais lattice, and $\mathbf{K}$ and $\mathbf{K}$ ' are the corners of the first Brillouin zone (Dirac Points).

$$
H_{0}(\mathbf{k})=t\left(\begin{array}{cc}
0 & 1+e^{-i \mathbf{k} \cdot \mathbf{T}_{2}}+e^{-i \mathbf{k} \cdot\left(\mathbf{T}_{2}-\mathbf{T}_{1}\right)} \\
1+e^{i \mathbf{k} \cdot \mathbf{T}_{2}}+e^{i \mathbf{k} \cdot\left(\mathbf{T}_{2}-\mathbf{T}_{1}\right)} & 0
\end{array}\right)
$$

The Hamiltonian is a $2 \times 2$ matrix because the honeycomb lattice is a Bravais lattice with a twoelement base. By diagonalizing this matrix we obtain the well-known energy bands of graphene

$$
E_{ \pm}(\mathbf{k})= \pm t \sqrt{2+2 \cos \left(\sqrt{3} k_{y} a\right)+4 \cos \left(\frac{\sqrt{3}}{2} k_{y} a\right) \cos \left(\frac{3}{2} k_{x} a\right)} .
$$

There is a cone-like dispersion relation of Dirac particles around the Dirac points (DP), which are the two non-equivalent corners of the first Brillouin zone, $\mathbf{K}$ and $\mathbf{K}$ '. This means that the low-energy excitations obey a conic dispersion relation and behave like Dirac fermions. Expressing $\mathbf{k}=\mathbf{K}+\left(q_{x}, q_{y}\right)$ with $\mathbf{K}$ one of the DP, and in the long-wavelength approximation $q_{x} a, q_{y} a \ll 1$, the Hamiltonian matrix takes the form

$$
H_{0}^{\mathbf{K}}(\mathbf{q})=\frac{3 t a}{2}\left(\begin{array}{cc}
0 & q_{x}+i q_{y} \\
q_{x}-i q_{y} & 0
\end{array}\right)
$$

which can be cast in the form of a 2D non-massive Dirac Hamiltonian,

$$
H_{0}^{\alpha}(\mathbf{q})=\hbar v_{F}\left(\alpha \sigma_{x} q_{x}-\sigma_{y} q_{y}\right)
$$

Here the Fermi velocity $v_{F}=3 a t / 2 \hbar$ is 300 times smaller than the speed of light, $\boldsymbol{\sigma}=\left(\sigma_{x}, \sigma_{y}\right)$ are the Pauli matrices, and we have associated the index $\alpha= \pm 1$ with each of the non-equivalent DP. The conic dispersion relation can be obtained by diagonalizing this matrix, being $E_{\mathbf{K}}(\mathbf{q})=$ 
$\pm \hbar v_{F} q$ near each DP. The eigenstates of these Hamiltonians are spinors, whose two components are associated with the two elements of the lattice base. The low-energy states in cylindrical coordinates for a circular graphene sheet of radius $r_{0}$ (we may assume that $r_{0} \rightarrow \infty$ at the end of the calculation) $\operatorname{are}^{25}-29$

$$
\Psi_{m, \nu, K}^{v}=\frac{1}{\sqrt{2}}\left(\begin{array}{c}
\psi_{m+1, \nu} \\
i \psi_{m, \nu}
\end{array}\right), \Psi_{m, \nu, K}^{c}=\frac{1}{\sqrt{2}}\left(\begin{array}{c}
\psi_{m+1, \nu} \\
-i \psi_{m, \nu}
\end{array}\right)
$$

near $\mathbf{K}$, and

$$
\Psi_{m, \nu, K^{\prime}}^{v}=\frac{1}{\sqrt{2}}\left(\begin{array}{c}
\psi_{m, \nu} \\
i \psi_{m+1, \nu}
\end{array}\right), \Psi_{m, \nu, K^{\prime}}^{c}=\frac{1}{\sqrt{2}}\left(\begin{array}{c}
\psi_{m, \nu} \\
-i \psi_{m+1, \nu}
\end{array}\right)
$$

near K', with

$$
\psi_{m, \nu}(r, \theta)=\frac{N_{m, \nu}}{2 \pi} J_{m}\left(q_{m, \nu} r\right) e^{i m \theta}
$$

where $N_{m, \nu}=\sqrt{2} / r_{0} J_{m+1}\left(x_{m, \nu}\right)$ is a normalization constant, $\{m, \nu\}$ are integers, and $q_{m, \nu}=$ $x_{m, \nu} / r_{0}$, where $x_{m, \nu}$ is the $\nu$ th zero of $J_{m}(x)$.

\section{A. Pseudospin, angular momentum, and helicity}

As is well known, the low-energy states of graphene are two-component spinors. These spinors should not be confused with the spin states of the electron (spin is not included in our tightbinding model): rather, they are related to the physical lattice. More precisely, each component is associated with the relative amplitude of the Bloch function in each sublattice of the honeycomb lattice. This degree of freedom is called pseudospin, for it plays a role in the Hamiltonian analogous to the one played by the regular spin in the Dirac Hamiltonian. It borrows the same $\mathrm{SU}(2)$ algebra but, unlike the isospin symmetry that connects protons and neutrons, pseudospin is actually an angular momentum, as has been shown by Mecklenburg and Regan $\underline{20}^{2}$ The common interpretation of this variable is that the pseudospin would be pointing up in $\hat{z}$ (outside the plane containing the graphene disk) in a state where all the electrons would be found in $A$ sites, while it would be pointing down in $\hat{z}$ if the electrons were located exclusively in the $B$ sublattice. Of course, in the eigenstates of the Hamiltonian, electrons are distributed homogeneously between the two sublattices, so the pseudospin is contained in the $x y$-plane. This fact can easily be corroborated for the states in Eqs. (5) and (66), since the mean value of the z-component of the operator associated with the pseudospin is identically zero for any of those states, $\left\langle m, \nu, \alpha\left|\sigma_{z}\right| m, \nu, \alpha\right\rangle=0$. 
Since our goal is to study the interaction of graphene with light carrying OAM, it is worth to examine the OAM of our electronic states. The z-component of the OAM operator is $L_{z}=-i \hbar(\partial / \partial \theta) \mathbb{I}$, where the identity operator acts on the pseudospin space. Since the effective Hamiltonian near one of the DP has circular symmetry [it depends only on the absolute value of $\left(q_{x}, q_{y}\right)$ ], one could naively expect the Hamiltonian to commute with the OAM. However, this is not the case:

$$
\left[H_{0}^{\alpha}, L_{z}\right]=-i \hbar v_{F} \alpha\left(\sigma_{x} p_{y}+\sigma_{y} p_{y}\right) .
$$

In order to construct a conserved angular momentum, we add the pseudospin to $L_{z}$, and define the total angular momentum as

$$
J_{z}^{\alpha}=L_{z}-\alpha \frac{\hbar}{2} \sigma_{z}
$$

It can be easily shown that this operator does commute with the Hamiltonian. In addition, our chosen basis states are eigenstates of this total angular momentum operator (near $\mathbf{K}$ ),

$$
J_{z} \Psi_{m, \nu, K}=\left(\begin{array}{c}
\hbar(m+1) \psi_{m+1, \nu} \\
\hbar m\left( \pm i \psi_{m, \nu}\right)
\end{array}\right)-\frac{\hbar}{2}\left(\begin{array}{c}
\psi_{m+1, \nu} \\
-( \pm i) \psi_{m, \nu}
\end{array}\right)=\left(m+\frac{1}{2}\right) \hbar \Psi_{m, \nu, K}
$$

In a similar way we found for states near $\mathbf{K}^{\prime}$ that their eigenvalue is also $j_{z}=(m+1 / 2) \hbar$. This means that for any state the total angular momentum is directly associated with the index $m$. In addition, since the mean value of the pseudospin is zero, the index $m$ can be associated with the mean value of the OAM too.

As an alternative interpretation, it is possible to see $L_{z}$ as an envelope angular momentum, an operator that acts only on the envelope-macroscopic - part of the wave function, as can be seen from its diagonal form in pseudospin space. The total angular momentum we have defined contains thus information on both the macroscopic and the microscopic (pseudospin) parts of the wavefunction.

There is another operator that commutes with $H_{0}^{\alpha}$, as in the regular Dirac equation: the helicity operator, defined near $\mathbf{K}$ as $\Sigma_{K}=(\hbar / 2) \boldsymbol{\sigma}^{*} \cdot \hat{q}$, where $\hat{q}=\left(q_{x}, q_{y}\right) /\left(q_{x}^{2}+q_{y}^{2}\right)^{1 / 2}$. The helicity is the component of the pseudospin in the direction of motion. The helicity is a constant of motion and is $+\hbar / 2$ for conduction-band states, and $-\hbar / 2$ for valence-band states, and it does not depend on the DP. The main advantage of this quantum number is that, unlike the total angular momentum, it is a constant of motion that differentiates the states in the conduction band from those in the valence band. 


\section{INTERACTION HAMILTONIAN}

We first consider the vector potential of the TL beam in the Coulomb gauge, given by

$$
\mathbf{A}(\mathbf{r}, t)=A_{0} e^{i\left(q_{z} z-\omega t\right)}\left[\boldsymbol{\epsilon}_{\sigma} J_{\ell}(q r) e^{i \ell \theta}-\sigma i \hat{\mathbf{z}} \frac{q}{q_{z}} J_{\ell+\sigma}(q r) e^{i(\ell+\sigma) \theta}\right]+c . c .,
$$

where $\boldsymbol{\epsilon}_{\sigma}=\hat{\mathbf{x}}+i \sigma \hat{\mathbf{y}}$ are the polarization vectors and $\sigma= \pm 1$. The radial profile of the beam is given by Bessel functions $J_{\ell}(q r)$ and $J_{\ell+\sigma}(q r)$. Alternatively, one can work with Laguerre-Gaussian modes instead $\frac{30}{\underline{3}}$ (A comment on notation: note that $q_{z}$ and $q$ refer to the light beam, while $q_{x}$, $q_{y}$, and $q_{m, \nu}$, to the electrons.) In order to describe the interaction between light and matter, we use the minimal-coupling Hamiltonian, obtained by the usual prescription $\mathbf{p} \rightarrow \mathbf{p}+e \mathbf{A}$ in Eq. (4), which guarantees local gauge invariance. By performing this substitution we get

$$
\begin{aligned}
H^{\alpha} & =\hbar v_{F}\left(\alpha \sigma_{x} q_{x}-\sigma_{y} q_{y}\right)+e v_{F}\left(\alpha \sigma_{x} A_{x}-\sigma_{y} A_{y}\right) \\
& \equiv H_{0}^{\alpha}+H_{\mathrm{int}}^{\alpha}
\end{aligned}
$$

where we have already found the solutions for $H_{0}^{\alpha}$ in polar coordinates, given by Eqs. (5) and (6) . Of course, the total Hamiltonian of the system is obtained by summing over $\alpha$ the Hamiltonian of Eq. (12). Since graphene is a two-dimensional system, only components $x$ and $y$ of the electromagnetic field appear, and hence its $z$ component is automatically eliminated from the interaction. Then, for a graphene disk placed at $z=0$, the vector potential (11) effectively becomes

$$
\mathbf{A}(r, \theta, t)=A_{0}(\hat{\mathbf{x}}+i \sigma \hat{\mathbf{y}}) e^{-i \omega t} J_{\ell}(q r) e^{i \ell \theta}+c . c .
$$

We define the following quantities

$$
\begin{aligned}
& A^{(+)}=A_{0} e^{-i \omega t} J_{\ell}(q r) e^{i \ell \theta}, \\
& A^{(-)}=A_{0} e^{i \omega t} J_{\ell}(q r) e^{-i \ell \theta},
\end{aligned}
$$

which can be associated with absorption and emission of one photon, respectively. Thus, we write the interaction Hamiltonian close to a Dirac point labelled by $\alpha$ and with a given polarization of the incoming light $\sigma$ as

$$
H_{\mathrm{int}}^{\alpha, \sigma}=e v_{F}\left(\begin{array}{cc}
0 & (\sigma-\alpha) A^{(+)}+(\alpha+\sigma) A^{(-)} \\
(\alpha+\sigma) A^{(+)}+(\alpha-\sigma) A^{(-)} & 0
\end{array}\right) .
$$

As we described in previous sections, the spinor components are associated with the two sublattices in the graphene structure. Thus, each interaction Hamiltonian $H_{\text {int }}^{\alpha, \sigma}$, having only off-diagonal 
elements, exchanges probability amplitude between the two sublattices. For example, for $\alpha=1$, i.e. near $\mathbf{K}$, and $\sigma=+1$ one obtains

$$
H_{\mathrm{int}}^{K,+}=2 e v_{F}\left(\begin{array}{cc}
0 & A^{(-)} \\
A^{(+)} & 0
\end{array}\right),
$$

and for $\alpha=-1$, i.e. near $\mathbf{K}^{\prime}$, and $\sigma=+1$ we have

$$
H_{\mathrm{int}}^{K^{\prime},+}=2 e v_{F}\left(\begin{array}{cc}
0 & A^{(+)} \\
A^{(-)} & 0
\end{array}\right) .
$$

The role of the individual matrix elements in these Hamiltonians becomes clearer when the rotatingwave approximation (RWA) applies. In the RWA, for a valence- to conduction-band transition, it is admisible to neglect $A^{(-)}$in Hamiltonians (16) and (17). Because of the resulting matrix form, it is clear that the action on an electron in a state close to $K$ is, in a sense, opossite to that on an electron in a state close to $K^{\prime}$. While in each Dirac point $H_{\text {int }}^{\alpha,+}$ exchanges pseudospin components, in $\mathrm{K}\left(\mathrm{K}^{\prime}\right)$ the up(down)-component is eliminated in the final resulting state. Then, from a microscopic point of view, an electron originally in a valence band state near a given Dirac point becomes "localized" in one sublattice.

\section{INTERACTION MATRIX ELEMENTS}

In order to calculate relevant observable quantities, we now obtain the light-matter interaction matrix elements for interband transitions. In the long-wavelength approximation we neglect the intervalley transitions, i.e. terms that connect states near different Dirac points. Hence, we need to calculate

$$
\left\langle c, m^{\prime}, \nu^{\prime}, \alpha\left|H_{\mathrm{int}}^{\alpha, \sigma}\right| v, m, \nu, \alpha\right\rangle=\int \Psi_{m^{\prime}, \nu^{\prime}, \alpha}^{c \dagger}(r, \theta) H_{\mathrm{int}}^{\alpha, \sigma}(r, \theta) \Psi_{m, \nu, \alpha}^{v}(r, \theta) d^{2} \mathbf{r} .
$$

Using the definition of $\Psi$, Eqs. (5) and (6), and the Hamiltonian, Eq. (15), we can write the matrix elements in the following way

$$
\begin{gathered}
\left\langle c, m^{\prime}, \nu^{\prime}, \alpha\left|H_{\mathrm{int}}^{\alpha,+}\right| v m, \nu, \alpha,\right\rangle=2 i e \alpha v_{F} \int\left(\psi_{m^{\prime}+1, \nu^{\prime}}^{*} A^{(-)} \Psi_{m, \nu}+\psi_{m^{\prime}, \nu^{\prime}}^{*} A^{(+)} \Psi_{m+1, \nu}\right) d \mathbf{r}, \\
\left\langle c, m^{\prime}, \nu^{\prime}, \alpha\left|H_{\mathrm{int}}^{\alpha,-}\right| v, m, \nu, \alpha\right\rangle=-2 i e \alpha v_{F} \int\left(\psi_{m^{\prime}, \nu^{\prime}}^{*} A^{(-)} \Psi_{m+1, \nu}+\psi_{m^{\prime}+1, \nu^{\prime}}^{*} A^{(+)} \Psi_{m, \nu}\right) d \mathbf{r} .
\end{gathered}
$$

Each matrix element contains only two terms, one associated with the absorption and the other with the emission of a photon. In the RWA and defining

$$
I_{m, \nu}^{m^{\prime}, \nu^{\prime}}=N_{m^{\prime} \nu^{\prime}} N_{m \nu} r_{0}^{2} \int_{0}^{1} y J_{m^{\prime}}\left(x_{m^{\prime} \nu^{\prime}} y\right) J_{\ell}\left(q r_{0} y\right) J_{m}\left(x_{m \nu} y\right) d y
$$


we obtain

$$
\begin{aligned}
& \left\langle c, m^{\prime}, \nu^{\prime}, \alpha\left|H_{\mathrm{int}}^{\alpha,+}\right| v, m, \nu, \alpha\right\rangle \cong 2 \operatorname{\alpha iev}_{F} A_{0} e^{-i \omega t} I_{m+1, \nu}^{m+1+\ell, \nu^{\prime}} \delta_{m^{\prime}, m+(\ell+1)}, \\
& \left\langle c, m^{\prime}, \nu^{\prime}, \alpha\left|H_{\mathrm{int}}^{\alpha,-}\right| v, m, \nu, \alpha\right\rangle \cong-2 i \alpha e v_{F} A_{0} e^{-i \omega t} I_{m, \nu}^{m+\ell, \nu^{\prime}} \delta_{m^{\prime}, m+(\ell-1)}, \\
& \left\langle v, m^{\prime}, \nu^{\prime}, \alpha\left|H_{\mathrm{int}}^{\alpha,+}\right| c, m, \nu, \alpha\right\rangle \cong-2 i \alpha e v_{F} A_{0} e^{i \omega t} I_{m, \nu}^{m-\ell, \nu^{\prime}} \delta_{m^{\prime}, m-(\ell+1)}, \\
& \left\langle v, m^{\prime}, \nu^{\prime}, \alpha\left|H_{\mathrm{int}}^{\alpha,-}\right| c, m, \nu, \alpha\right\rangle \cong 2 i \alpha e v_{F} A_{0} e^{i \omega t} I_{m+1, \nu}^{m+1-\ell, \nu^{\prime}} \delta_{m^{\prime}, m-(\ell-1)} .
\end{aligned}
$$

The transfer of angular momentum is signaled by the Kronecker's deltas, which relate the quantum numbers $m$ and $m^{\prime}$ of the initial and final states. This transferred angular momentum includes both OAM and spin. This is to be expected given that both bands in graphene have the same microscopic angular momentum ( $p$-type orbitals). In this sense interband transitions in graphene are analogous to intraband transitions in regular semiconductors, as shown previously for intraband transitions in quantum rings $\underline{13,14}$

\section{EQUATIONS OF MOTION FOR PHOTO-EXCITED ELECTRONS}

The graphene Hamiltonian in the absence of the electron-light interaction is

$$
\mathcal{H}_{0}^{\alpha}=\hbar v_{f} \sum_{m, \nu} q_{m, \nu}\left(\hat{a}_{c, m, \nu, \alpha}^{\alpha \dagger} \hat{a}_{c, m, \nu}^{\alpha}-\hat{a}_{v, m, \nu, \alpha}^{\alpha \dagger} \hat{a}_{v, m, \nu}^{\alpha}\right)
$$

where $\hat{a}_{\lambda, m, \nu}^{\alpha \dagger}\left(\hat{a}_{\lambda, m, \nu}^{\alpha}\right)$ are the creation (annihilation) operators for the electrons in the band tagged by the index $\lambda=c, v$ and in the valley $\alpha$. Considering only interband transitions, the electron-light interaction Hamiltonian is given by

$$
\begin{aligned}
\mathcal{H}_{\mathrm{int}}^{\alpha, \sigma} & =\sum_{m \nu, m^{\prime} \nu^{\prime}}\left(\left\langle c, m^{\prime}, \nu^{\prime}, \alpha\left|H_{\mathrm{int}}^{\alpha, \sigma}\right| v, m, \nu, \alpha\right\rangle \hat{a}_{c, m^{\prime}, \nu^{\prime}}^{\alpha \dagger} \hat{a}_{v, m, \nu}^{\alpha}\right. \\
& \left.+\left\langle v, m^{\prime}, \nu^{\prime}, \alpha\left|H_{\mathrm{int}}^{\alpha, \sigma}\right| c, m, \nu, \alpha\right\rangle \hat{a}_{v, m^{\prime}, \nu^{\prime}}^{\alpha \dagger} \hat{a}_{c, m, \nu}^{\alpha}\right) .
\end{aligned}
$$

In order to simplify the notation, hereafter we drop the $\alpha$ index whenever there is no ambiguity. The density matrix operator is $\hat{\rho}_{\lambda^{\prime}, m^{\prime}, \nu^{\prime} ; \lambda, m, \nu}=\hat{a}_{\lambda^{\prime}, m^{\prime}, \nu^{\prime}}^{\dagger} \hat{a}_{\lambda, m, \nu}$. The equation of motion for this operator in the Heisenberg picture is:

$$
i \hbar \frac{d}{d t} \hat{\rho}_{\lambda^{\prime}, m^{\prime}, \nu^{\prime} ; \lambda, m, \nu}=\left[\hat{\rho}_{\lambda^{\prime}, m^{\prime}, \nu^{\prime} ; \lambda, m, \nu}, \mathcal{H}^{\sigma}\right]
$$

This leads to the equations of motion for the density matrix operator, in an analogous way as has been shown in Ref. [12]. We consider the evolution of three types of operators: $\hat{\rho}_{c, m^{\prime}, \nu^{\prime} ; c, m, \nu}$, 
$\hat{\rho}_{v, m^{\prime}, \nu^{\prime} ; v, m, \nu}$, and $\hat{\rho}_{v, m^{\prime}, \nu^{\prime} ; c, m, \nu}$

$$
\begin{aligned}
i \hbar \frac{d}{d t} \hat{\rho}_{c, m^{\prime}, \nu^{\prime} ; c, m, \nu} & =\hbar v_{F}\left(q_{m, \nu}-q_{m^{\prime}, \nu^{\prime}}\right) \hat{\rho}_{c, m^{\prime}, \nu^{\prime} ; c, m, \nu} \\
& +\sum_{n, \mu}\left(\left\langle c, m, \nu\left|H_{\mathrm{int}}^{\sigma}\right| v, n, \mu\right\rangle \hat{\rho}_{c, m^{\prime}, \nu^{\prime} ; v, n, \mu}\right. \\
& \left.-\left\langle v, n, \mu\left|H_{\mathrm{int}}^{\sigma}\right| c, m^{\prime}, \nu^{\prime}\right\rangle \hat{\rho}_{v, n, \mu ; c, m, \nu}\right), \\
i \hbar \frac{d}{d t} \hat{\rho}_{v, m^{\prime}, \nu^{\prime} ; v, m, \nu} & =\hbar v_{F}\left(q_{m^{\prime}, \nu^{\prime}}-q_{m, \nu}\right) \hat{\rho}_{v, m^{\prime}, \nu^{\prime} ; v, m, \nu} \\
& +\sum_{n, \mu}\left(\left\langle v, m, \nu\left|H_{\mathrm{int}}^{\sigma}\right| c, n, \mu\right\rangle \hat{\rho}_{v, m^{\prime}, \nu^{\prime} ; c, n, \mu}\right. \\
& \left.-\left\langle c, n, \mu\left|H_{\mathrm{int}}^{\sigma}\right| v, m^{\prime}, \nu^{\prime}\right\rangle \hat{\rho}_{c, n, \mu ; v, m, \nu}\right), \\
i \hbar \frac{d}{d t} \hat{\rho}_{v, m^{\prime}, \nu^{\prime} ; c, m, \nu} & =\hbar v_{F}\left(q_{m^{\prime}, \nu^{\prime}}+q_{m, \nu}\right) \hat{\rho}_{v, m^{\prime}, \nu^{\prime} ; c, m, \nu} \\
& +\sum_{n, \mu}\left(\left\langle c, m, \nu\left|H_{\mathrm{int}}^{\sigma}\right| v, n, \mu\right\rangle \hat{\rho}_{v, m^{\prime}, \nu^{\prime} ; v, n, \mu}\right. \\
& \left.-\left\langle c, n, \mu\left|H_{\mathrm{int}}^{\sigma}\right| v, m^{\prime}, \nu^{\prime}\right\rangle \hat{\rho}_{c, n, \mu ; c, m, \nu}\right) .
\end{aligned}
$$

These equations are, of course, also valid for the expectation values of the density matrix operators, taken over the initial state of the graphene. We denote these expectation values $\rho_{c, m^{\prime}, \nu^{\prime} ; c, m, \nu}$, $\rho_{v, m^{\prime}, \nu^{\prime} ; v, m, \nu}$ (called populations when $m=m^{\prime}$ and $\nu=\nu^{\prime}$, and intraband quantum coherences otherwise) and $\rho_{v, m^{\prime}, \nu^{\prime} ; c, m, \nu}$ (interband coherences). Notice that in Eqs. (26)-(28) we keep the intraband coherences, which are essencial in the TL excitation process. These coherences are usually left out of the theory when the vertical-transition approximation is made.

\section{A. Low-excitation regime}

Due to the impossibility of solving analytically Eqs. (26)-(28) in all their generality, we will consider the case of low photoexcitation. In this case, an analytical perturbative approach is possible, which gives us the basic physical insight that we are looking for. We proceed in the following way, as in Ref. [12]: we first solve Eq. (28) for $\rho_{v, m^{\prime}, \nu^{\prime} ; c, m, \nu}$, considering that in the quasi-equilibrium and at temperatures much lower than the Fermi temperature for graphene, we

have $\rho_{v, m^{\prime}, \nu^{\prime} ; v, m, \nu}^{(0)}=\delta_{m, m^{\prime}} \delta_{\nu, \nu^{\prime}}$ and $\rho_{c, m^{\prime}, \nu^{\prime} ; c, m, \nu}^{(0)}=0$ (zeroth-order intraband elements). We then solve Eqs. (26) and (27) using the first-order solutions of Eq. (28). The equation of motion for the first-order interband polarization is

$$
\left[i \hbar \frac{d}{d t}-\hbar v_{F}\left(q_{m^{\prime}, \nu^{\prime}}+q_{m, \nu}\right)\right] \rho_{v, m^{\prime}, \nu^{\prime} ; c, m, \nu}^{(1)}=\left\langle c, m, \nu\left|H_{\mathrm{int}}^{\sigma}\right| v, m^{\prime}, \nu^{\prime}\right\rangle
$$


and its solution in the RWA, for a monochromatic electromagnetic field turned on at $t=0$, is given by

$$
\begin{aligned}
\rho_{v, m^{\prime}, \nu^{\prime} ; c, m, \nu}^{(1)} & =-\frac{1-e^{-i\left[v_{F}\left(q_{m, \nu}+q_{m^{\prime}, \nu^{\prime}}\right)-\omega\right] t}}{\hbar v_{F}\left(q_{m, \nu}+q_{m^{\prime}, \nu^{\prime}}\right)-\hbar \omega}\left\langle c, m, \nu\left|H_{\mathrm{int}}^{\sigma}\right| v, m^{\prime}, \nu^{\prime}\right\rangle \\
& \equiv Y_{m, \nu ; m^{\prime}, \nu^{\prime}}(t)\left\langle c, m, \nu\left|H_{\mathrm{int}}^{\sigma}\right| v, m^{\prime}, \nu^{\prime}\right\rangle .
\end{aligned}
$$

With this solution, we get the second order intraband coherence from Eqs. (26) and (27)

$$
\begin{aligned}
\rho_{v, m^{\prime}, \nu^{\prime} ; v, m, \nu}^{(2)}(t) & =\delta_{m^{\prime}, m} \delta_{\nu^{\prime}, \nu}-\frac{i}{\hbar} e^{-i v_{F}\left(q_{m^{\prime}, \nu^{\prime}}-q_{m, \nu}\right) t} \\
& \times \sum_{n, \mu}\left\langle v, m, \nu\left|H_{\mathrm{int}}^{\sigma}\right| c, n, \mu\right\rangle\left\langle c, n, \mu\left|H_{\mathrm{int}}^{\sigma}\right| v, m^{\prime}, \nu^{\prime}\right\rangle \\
& \times \int_{0}^{t} d t^{\prime} e^{i v_{F}\left(q_{m^{\prime}, \nu^{\prime}}-q_{m, \nu}\right) t}\left[Y_{n, \mu ; m^{\prime}, \nu^{\prime}}(t)-Y_{m, \nu ; n, \mu}^{*}(t)\right],
\end{aligned}
$$

for the valence band, and

$$
\begin{aligned}
\rho_{c, m^{\prime}, \nu^{\prime} ; c, m, \nu}^{(2)}(t) & =-\frac{i}{\hbar} e^{-i v_{F}\left(q_{m, \nu}-q_{m^{\prime}, \nu^{\prime}}\right) t} \\
& \times \sum_{n, \mu}\left\langle c, m, \nu\left|H_{\mathrm{int}}^{\sigma}\right| v, n, \mu\right\rangle\left\langle v, n, \mu\left|H_{\mathrm{int}}^{\sigma}\right| c, m^{\prime}, \nu^{\prime}\right\rangle \\
& \times \int_{0}^{t} d t^{\prime} e^{i v_{F}\left(q_{m, \nu}-q_{m^{\prime}, \nu^{\prime}}\right) t}\left[Y_{n, \mu ; m^{\prime}, \nu^{\prime}}^{*}(t)-Y_{m, \nu ; n, \mu}(t)\right] .
\end{aligned}
$$

for the conduction band. As an example, we show the populations for a TL beam with right-handed circular polarization:

$$
\begin{aligned}
& n_{v, m, \nu}^{(2)}(t)=1-\frac{8 e^{2} v_{F}^{2} A_{0}^{2}}{\hbar^{2}} \sum_{\mu} \frac{\left(I_{m+1, \nu}^{m+1+\ell, \mu}\right)^{2}\left\{1-\cos \left[\left(v_{F}\left(q_{m, \nu}+q_{m+(\ell+1), \mu}\right)-\omega\right) t\right]\right\}}{\left[v_{F}\left(q_{m, \nu}+q_{m+(\ell+1), \mu}\right)-\omega\right]^{2}} \\
& n_{c, m, \nu}^{(2)}(t)=\frac{8 e^{2} v_{F}^{2} A_{0}^{2}}{\hbar^{2}} \sum_{\mu} \frac{\left(I_{m, \nu}^{m-\ell, \mu}\right)^{2}\left\{1-\cos \left[\left(v_{F}\left(q_{m, \nu}+q_{m-(\ell+1), \mu}\right)-\omega\right) t\right]\right\}}{\left[v_{F}\left(q_{m, \nu}+q_{m-(\ell+1), \mu}\right)-\omega\right]^{2}} .
\end{aligned}
$$

Near resonance, the populations evolve slowly, as expected. Also, the angular momentum of the intermediate state differs from that of the initial state by $\ell \pm 1$, the total angular momentum of the photon.

The populations' functional forms reflect, in part, what is expected based on general ideas of optical excitation. However, they are affected by the parameter dependence of the integral $I_{m, \nu}^{m^{\prime}, \nu^{\prime}}$, which is proportional to the light-matter matrix elements and carries information about the twisted character of the electromagnetic field. Therefore, to gain more insight into the population kinetics, we calculate [using Eq. (21)] and discuss some examples of the integrals $I_{m, \nu}^{m-\ell, \mu}$, which contribute to the population of the conduction-band states according to Eq. (34). We start by fixing the 
system (TL beam and graphene disk) parameters $\ell$ and $q \cdot r_{0}$ and inspect which matrix elements are relevant for the population of a state $(c, m, \nu)$ of the conduction band. Since $I_{m, \nu}^{m-\ell, \mu}$ enters the population through a sum over its $\mu$ parameter, in Fig. 2 we plot three different curves of $\left|I_{m, \nu}^{m-\ell}\right|$, for $\nu=40,60,80$, as a funcion of $\mu$ (with $\ell=10, q \cdot r_{0}=10$, and $m=20$ ). For this choice of parameters, we notice that the curves have a fairly well-behaved bell shape, and that the peak value occurs close to $\mu=\nu$, slightly shifted to the right. By plotting similar curves for various values of $m$, one can easily conclude that they are mainly insensitive to the value of $m$.

Now we can vary the light-beam and graphene disk parameters. In Fig. 3, we plot $\left|I_{m, \nu}^{m-\ell, \mu}\right|$ for $\ell=5,10,12,20$ as a funcion of $\mu$ (with $q \cdot r_{0}=10, \mu=60$, and $m=20$ ). We notice that the curves have a well-defined bell shape for the larger values of $\ell$, but not for $\ell=5$. Furthermore, for the former cases, we see that the bell widens as $\ell$ increases, and at the same time, it rapidly decreases in amplitude. For (small) $\ell=5$, the nice bell shape is lost and many values of $\mu$ contribute to the population with similar weight. All the cases without a sharp bell-like curve share an important characteristic of the population kinetics: many valence-band states contribute to the population of a given conduction-band state, and therefore a clear picture of Bloch oscillations is lost. Finally, we vary the parameter $q \cdot r_{0}$, which combines the radial dependence of the beam with the radius of the graphene disk. A large value of $q . r_{0}$ means that the light field makes many oscillations in the radial direction inside the material disk. In Fig. (4), we plot $\left|I_{m, \nu}^{m-\ell, \mu}\right|$ for $q \cdot r_{0}=7,10,20$ as a funcion of $\mu$ (with $\ell=10, \mu=60$, and $m=20$ ). It is seen that for $q \cdot r_{0}=7,10$ one obtains a clean bell shape, while for $q \cdot r_{0}=20$ the picture is similar to that of the $\ell=5$ (and q. $r_{0}=10$ ) case in Fig. 3. We interpret that this kind of behavior appears whenever $q . r_{0}>\ell$ and therefore the light field "oscillates more" in the radial coordinate than in the angular one, in which case even an approximate selection rule for the quantum number $\nu$ is lost.

\section{EVOLUTION OF PHYSICAL MAGNITUDES}

The modification of the electronic state of graphene by twisted light can be described in terms of the evolution of key physical quantities. In this Section we find the expressions for the photocurrents and the orbital angular momentum, using the lowest-order contributions to the density matrix obtained above. 


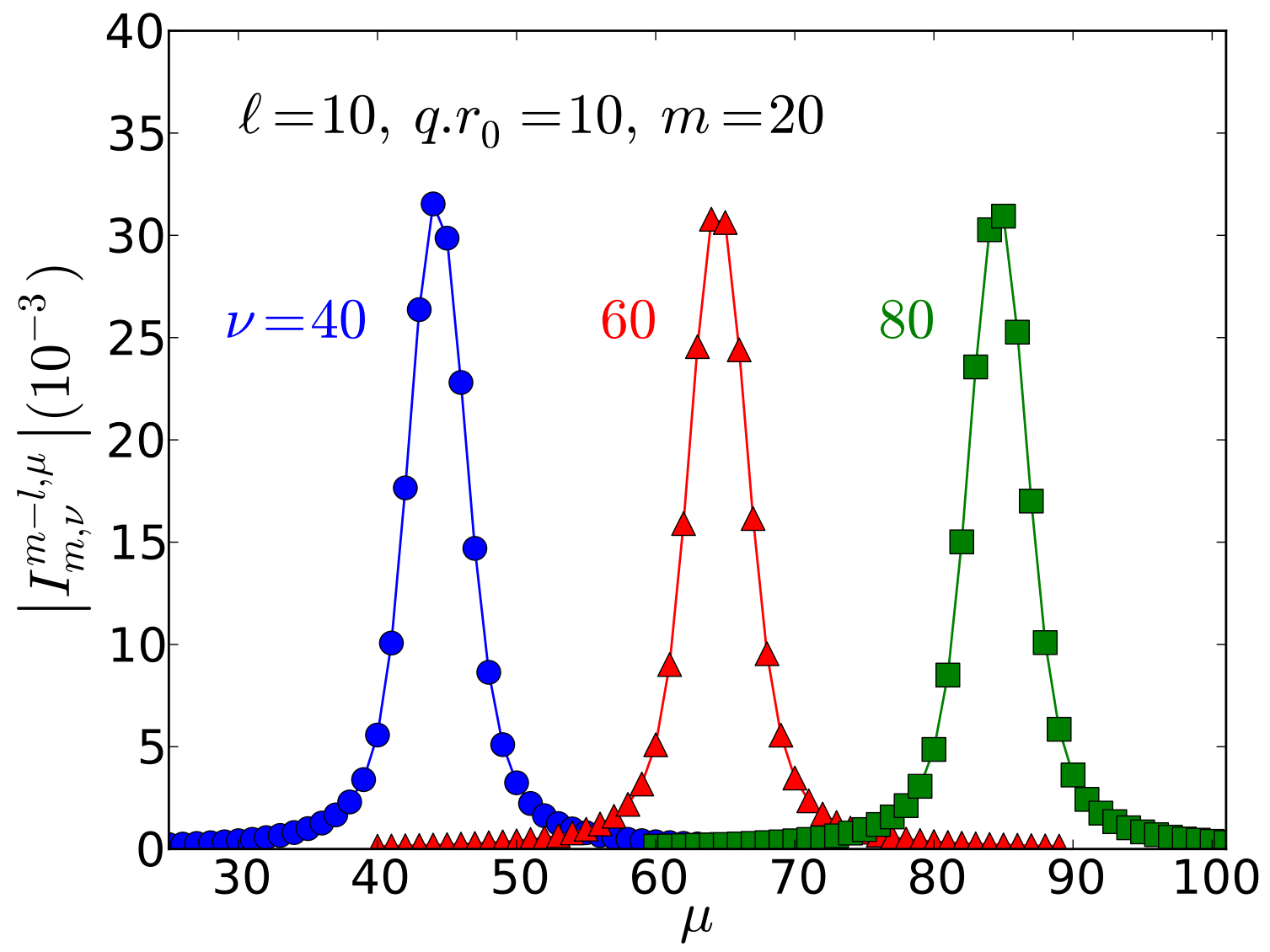

FIG. 2: Absolute value of the integral proportional to the matrix elements of the interaction of twisted light with graphene, which determines the population of the conduction-band excited states [see Eqs. (21) and [34)], for three different values of the quantum number $\nu$ of the final state.

\section{A. Transfer of angular momentum}

In Sec. 【IA we defined operators for the orbital and total angular momentum. In second quantization formalism and taking the expectation value over the ground state of graphene, the total angular momentum, which is diagonal in our basis, is given by the simple expression

$$
J_{z}(t)=2 \sum_{\lambda, m, \nu}\left(m+\frac{1}{2}\right) n_{\lambda, m, \nu}^{(2)}(t),
$$

where the factor 2 comes from the valley degeneracy. $J_{z}(t)$ depends only on the populations, and thus evolves slowly with a magnitude proportional to amplitude of the electromagnetic field squared. 


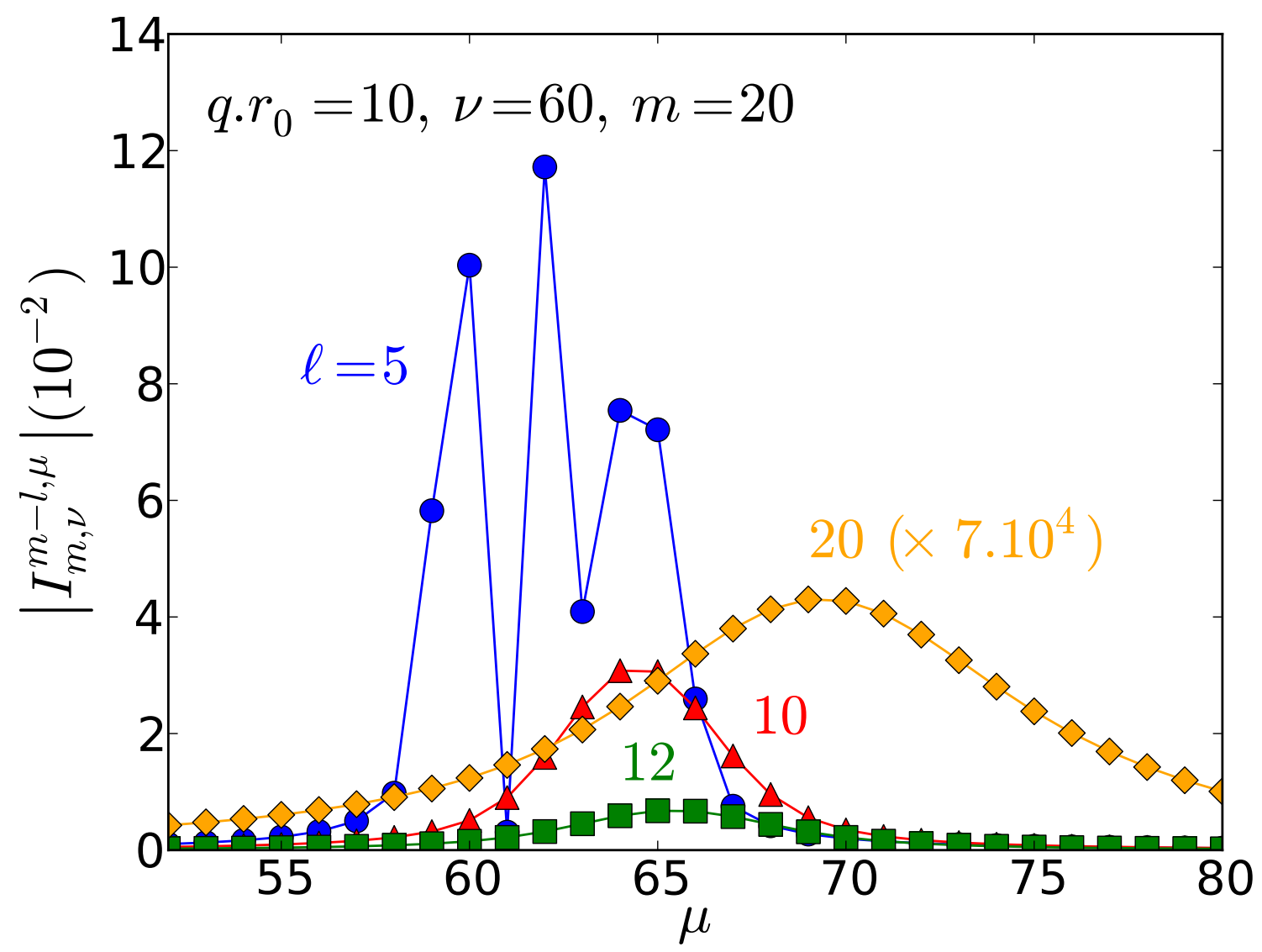

FIG. 3: Same as Fig. 2, for four different values of the twisted-light beam's orbital angular momentum, $\ell$.

On the other hand, we get for the OAM in operator form a slightly more complicated expression

$$
\begin{aligned}
L_{z}(t) & =\sum_{\alpha, m, \nu}\left(m+\frac{1}{2}\right) \hbar\left[n_{c, m, \nu}^{\alpha(2)}(t)+n_{v, m, \nu}^{\alpha(2)}(t)\right] \\
& +\frac{\hbar}{2} \sum_{m \nu} \sum_{\lambda \neq \lambda^{\prime}}\left[\rho_{\lambda^{\prime}, m, \nu ; \lambda, m, \nu}^{K(1)}(t)-\rho_{\lambda^{\prime}, m, \nu ; \lambda, m, \nu}^{K^{\prime}(1)}(t)\right] .
\end{aligned}
$$

However, this expression greatly simplifies when we take the expectation value over an initial state, for $\rho_{\lambda^{\prime} m \nu, \lambda m \nu}^{K(1)} \propto \delta_{m, m+(\ell \pm 1)}=0$. Then, the expectation value of the OAM coincides with that of the total angular momentum.

\section{B. Diamagnetic contribution to the angular momentum}

In Sec. III, we included the light field by the substitution $\mathbf{p} \rightarrow \mathbf{p}+e \mathbf{A}$. In the angular momentum operator, this substitution introduces the so-called diamagnetic term $L_{z}^{(d i a)}=e(\mathbf{r} \times \mathbf{A})_{z}$, which we 


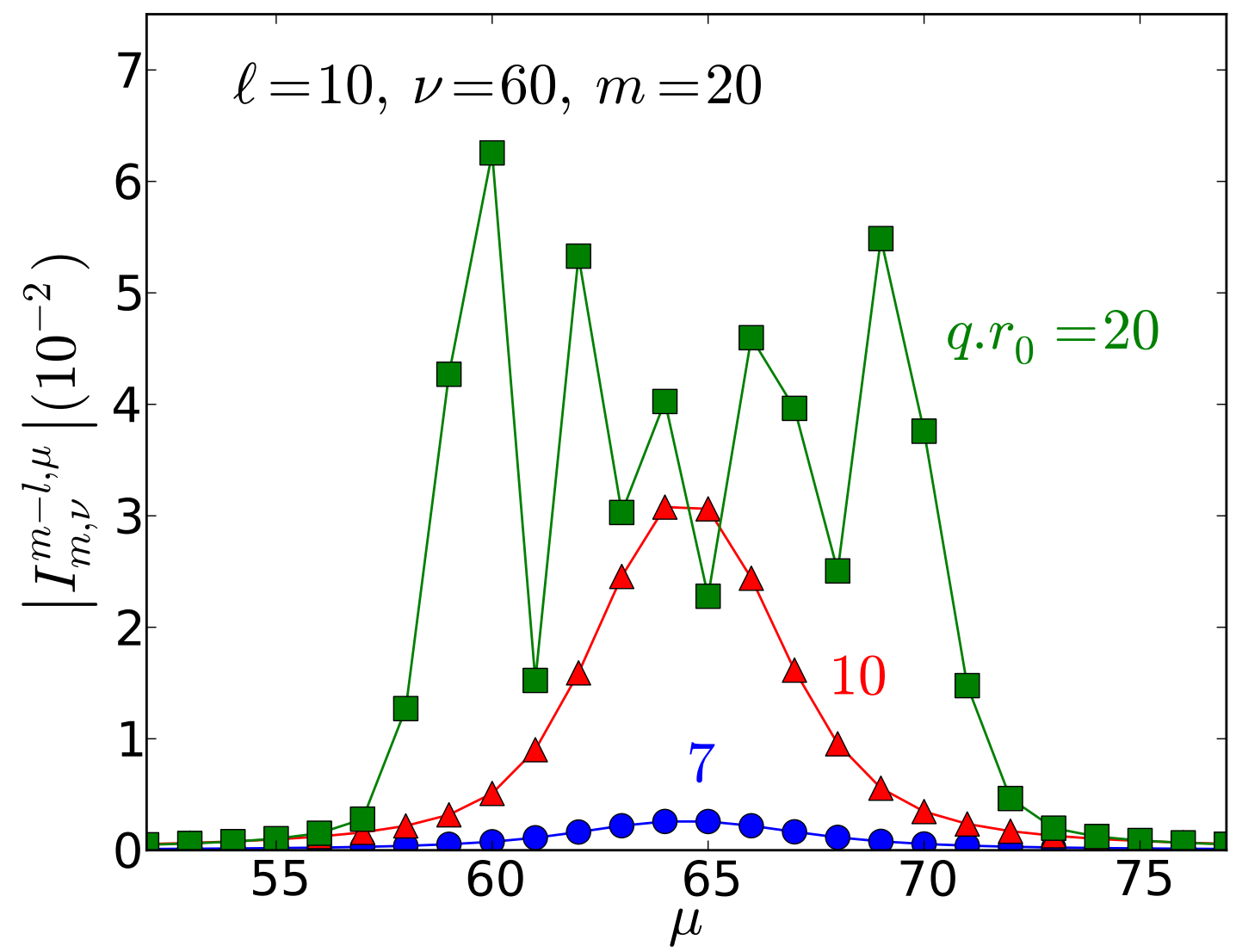

FIG. 4: Same as Fig. 2] for three different values of the system (twisted-light beam and graphene disk) parameter $q \cdot r_{0}$.

now consider. Its single-particle form is

$$
L_{z}^{(d i a)}=e i \sigma A_{0} r J_{\ell}(q r)\left[e^{-i \omega t} e^{i(\ell+\sigma) \theta}-e^{i \omega t} e^{-i(\ell+\sigma) \theta}\right] .
$$

This operator is of first-order in $A_{0}$. The expectation value of any operator can be expressed, in our scheme, as a sum of terms proportional to the populations and coherences, each multiplied by the appropriate matrix element of the single-particle operator. Since populations are of secondorder in $A_{0}$, the corresponding term in $\left\langle L_{z}^{(d i a)}\right\rangle$ is proportional to $A_{0}^{3}$ and is thus neglected in our second-order, low-excitation treatment. Instead, the coherence terms in $\left\langle L_{z}^{(d i a)}\right\rangle$ are of order $A_{0}^{2}$, and should be kept. The latter terms are especially relevant since the coherence terms of the paramagnetic angular momentum, which would be of first order, actually vanish here (see Sect. 
VIA). The matrix elements of $L_{z}^{(d i a)}$ are given by

$$
\begin{aligned}
\left\langle\lambda^{\prime}, m^{\prime}, \nu^{\prime}, K\left|L_{z}^{(d i a)}\right| \lambda, m, \nu, K\right\rangle & =e i \sigma A_{0}\left[\delta_{m^{\prime}, m+(\ell+\sigma)} e^{-i \omega t}-\delta_{m^{\prime}, m-(\ell+\sigma)} e^{i \omega t}\right] \times \\
& \times \int\left[J_{m^{\prime}+1, \nu^{\prime}}\left(q_{m^{\prime}+1, \nu^{\prime}} r\right) J_{m+1, \nu}\left(q_{m+1, \nu} r\right) J_{\ell}(q r)\right. \\
& \left.-J_{m^{\prime}, \nu^{\prime}}\left(q_{m^{\prime}, \nu^{\prime}} r\right) J_{m, \nu}\left(q_{m, \nu} r\right) J_{\ell}(q r)\right] r^{2} d r .
\end{aligned}
$$

The second-quantized form of $L_{z}^{(d i a)}$ requires a summation over all possible values of $m, m^{\prime}, \nu$ and $\nu^{\prime}$. The summation over $m^{\prime}$ results in only one term, and the summation over $m^{\prime}$ can be thought in the following way:

$$
\begin{aligned}
& \sum_{\forall m} \int d r r^{2}\left[J_{m \pm(\ell+\sigma)+1, \nu^{\prime}}\left(q_{m \pm(\ell+\sigma)+1, \nu^{\prime}} r\right) J_{m+1, \nu}\left(q_{m+1, \nu} r\right) J_{\ell}(q r)\right] \\
& \approx \sum_{\forall m} \int d r r^{2}\left[J_{m \pm(\ell+\sigma), \nu^{\prime}}\left(q_{m \pm(\ell+\sigma)+1, \nu^{\prime}} r\right) J_{m, \nu}\left(q_{m, \nu} r\right) J_{\ell}(q r)\right] .
\end{aligned}
$$

This expression is only approximately accurate, since we are not actually summing over all $m$, but the two members of Eq. (40) differ only in two terms, and we will neglect them in this work.

\section{Induced photocurrents}

In this Section we obtain the photoinduced currents produced by the irradiation with TL. Their first-quantized form is obtained by writing the interaction Hamiltonian as $H_{\mathrm{int}}^{\alpha}=-\boldsymbol{\jmath}^{\alpha} \cdot \mathbf{A}$, thus:

$$
\boldsymbol{j}^{\alpha}=-e v_{F}\left(\alpha \sigma_{x},-\sigma_{y}\right) .
$$

In second quantization notation, the density current will be given by:

$$
\boldsymbol{J}^{\alpha}=e v_{F} \hat{\Psi}^{\alpha \dagger}(\mathbf{r}, t)\left(-\alpha \sigma_{x}, \sigma_{y}\right) \hat{\Psi}^{\alpha}(\mathbf{r}, t)
$$

where $\hat{\Psi}^{\alpha}$ are the field operators of the system. This result is consistent with the current obtained from the Dirac Hamiltonian. Given the symmetries of this problem, and in order to find a relation between the induced currents and the transfer of angular momentum, we write the currents in cylindrical coordinates, and separate its $\hat{r}$ and $\hat{\theta}$ components. By writing the field operators in terms of creation and annihilation operators, we get

$$
\begin{gathered}
J_{r}^{\alpha}(\mathbf{r}, t)=-e v_{F} \sum_{\lambda, \lambda^{\prime}} \sum_{m, m^{\prime}} \sum_{\nu, \nu^{\prime}} \Psi_{\lambda, m, \nu}^{\alpha \dagger}(\mathbf{r})\left(\alpha \sigma_{x} \cos \theta-\sigma_{y} \sin \theta\right) \Psi_{\lambda^{\prime}, m^{\prime}, \nu^{\prime}}^{\alpha}(\mathbf{r}) \rho_{\lambda, m, \nu ; \lambda^{\prime}, m^{\prime}, \nu^{\prime}}^{\alpha}(t), \\
J_{\theta}^{\alpha}(\mathbf{r}, t)=-e v_{F} \sum_{\lambda, \lambda^{\prime}} \sum_{m, m^{\prime}} \sum_{\nu, \nu^{\prime}} \Psi_{\lambda, m, \nu}^{\alpha \dagger}(\mathbf{r})\left(-\alpha \sigma_{x} \sin \theta-\sigma_{y} \cos \theta\right) \Psi_{\lambda^{\prime}, m^{\prime}, \nu^{\prime}}^{\alpha}(\mathbf{r}) \rho_{\lambda, m, \nu ; \lambda^{\prime}, m^{\prime}, \nu^{\prime}}^{\alpha}(t) .
\end{gathered}
$$


We next write separately the interband coherence contribution from the intraband coherence and population contributions.

An expression for the interband coherence contribution can be obtained using the functional form of $\rho_{v, m, \nu ; c, m^{\prime}, \nu^{\prime}}^{\alpha(1)}[$ Eq. (30)] and the matrix elements in the RWA [Eq. (22) ]. We show the results for left-handed polarized light $(\sigma=1)$. The $\hat{r}$ component reads

$$
\begin{aligned}
& J_{r}^{\alpha(c o h)}(\mathbf{r}, t)=-4 A_{0} e^{2} v_{F}^{2} \cos [(\ell+1) \theta] \\
& \times \sum_{m, \nu, \nu^{\prime}} \frac{N_{m+1, \nu} N_{m+\ell+1, \nu^{\prime}}}{2 \pi} J_{m+1}\left(q_{m+1, \nu} r\right) J_{m+\ell+1}\left(q_{m+\ell+1, \nu^{\prime}} r\right) \\
& \times I_{m+1, \nu}^{m+1+\ell, \nu^{\prime}}(q, \ell)\left[e^{-i \omega t} Y_{(m+\ell+1), \nu^{\prime} ; m, \nu}(t)+e^{i \omega t} Y_{(m+\ell+1), \nu^{\prime} ; m, \nu}^{*}(t)\right] .
\end{aligned}
$$

For the $\hat{\theta}$ component we get

$$
\begin{aligned}
& J_{\theta}^{\alpha(c o h)}(\mathbf{r}, t)=4 A_{0} e^{2} v_{F}^{2} \sin [(\ell+1) \theta] \\
& \times \sum_{m, \nu, \nu^{\prime}} \frac{N_{m+1, \nu} N_{m+\ell+1, \nu^{\prime}}}{2 \pi} J_{m+1}\left(q_{m+1, \nu} r\right) J_{m+\ell+1}\left(q_{m+\ell+1, \nu^{\prime}} r\right) \\
& \times I_{m+1, \nu}^{m+1+\ell, \nu^{\prime}}(q, \ell)\left[e^{-i \omega t} Y_{(m+\ell+1), \nu^{\prime} ; m, \nu}(t)+e^{i \omega t} Y_{(m+\ell+1), \nu^{\prime} ; m, \nu}^{*}(t)\right] .
\end{aligned}
$$

We note that the $\hat{\theta}$ component is identically zero when $\ell=-\sigma$, which shows that if the photon carries no total angular momentum the induced currents do not rotate around the beam's axis. On the other hand, the $\hat{r}$ component remains finite for all values of $\ell$.

For the population and intraband coherences, the lowest non-trivial order does not depend on the valley. Noticing that $\rho_{\lambda, m, \nu ; \lambda, m^{\prime}, \nu^{\prime}}^{(2)} \propto \delta_{m, m^{\prime}}$ for all $\lambda, \nu, \nu^{\prime}$, the $\hat{r}$ component will be identically zero at second order in $A_{0}$, since

$$
J_{r}^{(p o p)}(\mathbf{r}, t) \propto \sin \left[\left(m^{\prime}-m\right) \theta\right] \delta_{m^{\prime}, m}=0,
$$

and the $\hat{\theta}$ component does not depend on the azimuthal coordinate, and is given by

$$
J_{\theta}^{(p o p)}(\mathbf{r}, t)=2 e v_{F} \sum_{m, \nu, \nu^{\prime}} \frac{N_{m+1, \nu} N_{m, \nu^{\prime}}}{2 \pi} J_{m+1}\left(q_{m+1, \nu} r\right) J_{m}\left(q_{m, \nu^{\prime}} r\right)\left(\rho_{v, m, \nu ; v, m, \nu^{\prime}}^{(2)}(t)-\rho_{c, m, \nu ; c, m, \nu^{\prime}}^{(2)}(t)\right) .
$$

Here, the OAM of the light, $\ell$, appears implicitely through the population and intraband coherences [see Eqs. (33) and (34)]. Finally, its worth mentioning that even in the case with $\ell=0$ (i.e. when the light does not carry OAM and it is just a regular beam with a radial Bessel dependence instead of Gaussian), there still is a transfer of angular momentum to the electrons, evidenced by a photocurrent that rotates around the beam's axis. This can be seen as a transfer of spin 
angular momentum of the photons to the orbital angular momentum of the electrons. This does not happen in the case of interband transitions in semiconductors, $\frac{12}{2}$ where the matrix elements are proportional to $\delta_{m^{\prime}, m \pm \ell}$. Our result, however, agrees with the findings of the study of intraband transitions in semiconductors, for the reasons explained in a previous section.

\section{CONCLUSIONS}

We studied theoretically the interaction of graphene with twisted light. As a starting point, using the tight-binding states of electrons in graphene near the Dirac points, we determined the light-matter interaction matrix elements.

In the RWA, for a valence- to conduction-band transition, we found that the action of the light-matter Hamiltonian on an electron in a state close to $K$ is, in a sense, opposite to that on an electron in a state close to $K^{\prime}$. The pseudospin components are exchanged in each Dirac point, and in addition, in $K\left(K^{\prime}\right)$ the up(down)-component is eliminated in the final resulting state.

We examined the dynamics of the photoexcitation process by posing the equations of motion of the density matrix, and obtaining the time evolution of the angular momentum of the photoexcited electrons and their associated photocurrents. The transfer of spin and orbital angular momentum from light to the electrons, is more akin here to the case of intraband than of interband transitions in semiconductors. This is due to the fact that the two relevant energy bands of graphene originate from the same atomic orbitals.

\section{Acknowledgments}

We acknowledge financial support from the University of Buenos Aires through the program UBACYT 2011-2014 and Beca Estímulo for M.B.F. Figures_2, 3, and4were made with the graphics package matplotlib $\underline{\underline{31}}$

1 A. H. Castro Neto, F. Guinea, N. M. R. Peres, K. S. Novoselov, and A. K. Geim, Rev. Mod. Phys. 81, 109 (2009).

2 D. S. L. Abergel, V. Apalkov, J. Berashevich, K. Zieglerc, and T. Chakraborty, Adv. Phys. 59, 261 (2010).

3 K. S. Novoselov, A. K. Geim, S. V. Morozov, D. Jiang, M. I. Katsnelson, I. V. Grigorieva, S. V. Dubonos, and A. A. Firsov, Nature 438, 197 (2005). 
4 N. Stander, B. Huard, and D. Goldhaber-Gordon, Phys. Rev. Lett. 102, 026807 (2009).

5 A. F. Young and P. Kim, Nat. Phys. 8, 222 (2009).

6 A. Kuzmenko, E. van Heumen, F. Carbone, D. van der Marel, Phys. Rev. Lett. 100, 117401 (2008).

7 E. Nair, P. Blake, A. Grigorenko, K. Novoselov, T. Booth, T. Stauber, N. Peres, and A. Geim, Science 320, 308 (2008).

8 L. Allen, M. W. Beijersbergen, R. J. C. Spreeuw, and J. P. Woerdman, Phys. Rev. A 45, 8185 (1992).

9 D. L. Andrews, Structured Light and Its Applications: An Introduction to Phase-Structured Beams and Nanoscale Optical Forces (Academic Press, 2008).

10 G. F. Quinteiro and P. I. Tamborenea, EPL 85, 47001 (2009).

11 G. F. Quinteiro and P. I. Tamborenea, Phys. Rev. B 79, 155450 (2009).

12 G. F. Quinteiro and P. I. Tamborenea, Phys. Rev. B 82, 125207 (2010).

13 G. F. Quinteiro and J. Berakdar, Opt. Express 17, 20465 (2009).

14 G. F. Quinteiro, P. I. Tamborenea, and J. Berakdar, Opt. Express 19, 26733 (2011).

15 M. F. Andersen, C. Ryu, P. Clade, V. Natarajan, A. Vaziri, K. Helmerson, and W. D. Phillips, Phys. Rev. Lett. 97, 170406 (2006).

16 J. Watzel, A. S. Moskalenko, and J. Berakdar, Opt. Express 20, 27792 (2012).

17 Y. Ueno, Y. Toda, S. Adachi, R. Morita, and T. Tawara, Opt. Express 17, 20567 (2009).

18 N. B. Clayburn, J. L. McCarter, J. M. Dreiling, M. Poelker, D. M. Ryan, and T. J. Gay, Phys. Rev. B 87, $035204(2013)$

19 E. J. Mele, P. Kral, and D. Tomanek, Phys. Rev. B 61, 7669 (2000).

20 M. Mecklenburg and B. C. Regan, Phys. Rev. Lett. 106, 116803 (2011).

21 W. Schäfer and M. Wegener, Semiconductor Optics and Transport Phenomena (Springer, New York, 2002).

22 T. Winzer, A. Knorr, and E. Malic, Nano Lett. 10, 4839 (2010).

23 A. Ferreira, J. Viana-Gomes, Y. V. Bludov, V. M. Pereira, N. M. R. Peres, and A. H. Castro Neto, Phys. Rev. B 84, 235410 (2011).

24 A. Ferreira, N. M. R. Peres, R. M. Ribeiro, and T. Stauber, Phys. Rev. B 85, 115438 (2012).

25 P. Recher, B. Trauzettel, A. Rycerz, Ya. M. Blanter, C. W. J. Beenakker, and A. F. Morpurgo, Phys. Rev. B 76, 235404 (2007).

26 M. Hentschel and F. Guinea, Phys. Rev. B 76, 115407 (2007).

27 B. Wunsch, T. Stauber, and F. Guinea, Phys. Rev. B 77, 035316 (2008).

28 J. Cserti, A. Pályi, and C. Péterfalvi, Phys. Rev. Lett. 99, 246801 (2007).

29 N. M. R. Peres, J. N. B. Rodrigues, T. Stauber, and J. M. B. Lopes dos Santos, J. Phys.: Condens. Matter 21, 344202, (2009).

30 L. C. Dávila-Romero, D. L. Andrews, and M. Babiker, J. Opt. B: Quantum Semiclassical Opt. 4, S66 (2002).

31 J. D. Hunter, Comput. Sci. Eng. 9, 90 (2007). 